Article

\title{
Unconventional Transport Properties of Reduced Tungsten Oxide $\mathrm{WO}_{2.9}$
}

\author{
Alexander Shengelaya ${ }^{1,2, *}$, Fabio La Mattina ${ }^{3}$ and Kazimierz Conder 4 \\ 1 Department of Physics, Ivane Javakhishvili Tbilisi State University, Chavchavadze 3, GE-0128 Tbilisi, Georgia \\ 2 Andronikashvili Institute of Physics, Ivane Javakhishvili Tbilisi State University, 0177 Tbilisi, Georgia \\ 3 Laboratory for Transport at Nanoscale Interfaces, Empa Swiss Federal Laboratories for Science and \\ Technology, 8600 Dübendorf, Switzerland; Fabio.Lamattina@empa.ch \\ 4 Laboratory for Multiscale Materials Experiments, Paul Scherrer Institute, 5232 Villigen PSI, Switzerland; \\ kazimierz.conder@psi.ch \\ * Correspondence: alexander.shengelaya@tsu.ge
}

Received: 12 September 2020; Accepted: 14 October 2020; Published: 16 October 2020

\begin{abstract}
The temperature and magnetic field dependence of resistivity in $\mathrm{WO}_{2.9}$ was investigated. The variation of resistivity with temperature displayed unusual features, such as a broad maximum around $230 \mathrm{~K}$ and a logarithmic increase of resistivity below $16 \mathrm{~K}$. In the temperature range 16-230 K, we observed metallic-like behavior with a positive temperature coefficient. The combined analysis of resistivity and magnetoresistance (MR) data shows that these unusual transport properties of $\mathrm{WO}_{2.9}$ can be understood by considering the (bi)polaronic nature of charge carriers. In contrast to magnetization data, superconducting transition below $T_{\mathrm{C}}=80 \mathrm{~K}$ was not detected in resistivity measurements, indicating that the superconductivity is localized in small regions that do not percolate. We found a strong increase in positive MR below $80 \mathrm{~K}$. This effect is similar to that observed in underdoped cuprates, where the substantial increase of MR is attributed to superconducting fluctuations in small clusters. Therefore, the temperature dependence of MR indicates the presence of non-percolating superconducting clusters in $\mathrm{WO}_{2.9}$ below $80 \mathrm{~K}$ in agreement with magnetization data.
\end{abstract}

Keywords: tungsten oxide; polarons; superconductivity

\section{Preface}

The authors of this paper have been fortunate and privileged to collaborate with K. Alex Müller over many years. This collaboration still continues and in this Special Issue of the journal dedicated to his life's work, we would like to present the recent results of a project that was initiated and supported by Alex. Since the beginning of his illustrious scientific carrier, he has investigated a remarkable variety of physical properties of oxide perovskites, and especially $\mathrm{SrTiO}_{3}$. This research culminated in the discovery of high- $T_{\mathrm{C}}$ superconductivity in copper oxides. We are grateful to Alex for uncovering the fascinating world of the perovskite oxides, which continue to attract the attention of condensed matter physicists and materials scientists for more than 50 years.

\section{Introduction}

It is remarkable that reduced $\mathrm{SrTiO}_{3-\mathrm{x}}$ was the first oxide in which superconductivity was found in 1964 [1]. Only nine months after the $\mathrm{SrTiO}_{3}$ discovery, superconductivity was also reported for another perovskite oxide, sodium-doped $\mathrm{WO}_{3}$ [2]. Both $\mathrm{SrTiO}_{3-\mathrm{x}}$ and $\mathrm{Na}_{\mathrm{x}} \mathrm{WO}_{3}$ have a low $T_{\mathrm{c}}$ below $1 \mathrm{~K}$. However, superconducting regions with unusually high critical temperature $\left(T_{\mathrm{C}}=90 \mathrm{~K}\right)$ were observed on the surface of $\mathrm{Na}$-doped $\mathrm{WO}_{3}$ crystals [3,4]. Unfortunately, the composition of the superconducting regions could not be determined due to their small size. Another possible method to dope charge 
carriers in a tungsten oxide is to reduce the oxygen content. Filamentary superconductivity below $T_{\mathrm{c}}=3 \mathrm{~K}$ was observed in twin walls of reduced $\mathrm{WO}_{3-\mathrm{x}}$ single crystals [5]. Despite the filamentary characteristics of superconductivity and low $T_{c}$, an unusually large upper critical field $\mu_{0} H_{c 2}(0)$ above $15 \mathrm{~T}$ was found in $\mathrm{WO}_{3-\mathrm{x}}$. This large $\mu_{0} \mathrm{H}_{c 2}(0)$ violates the Pauli paramagnetic limit by a factor of three. In our opinion, this interesting finding deserves further study.

The parent compound $\mathrm{WO}_{3}$ has a perovskite-like structure with a vacant $\mathrm{A}$ site and consists of a

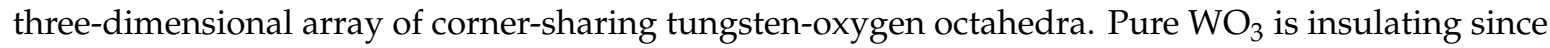
the $\mathrm{W}^{6+}\left(5 d^{0}\right)$ has an empty d-shell. Creating oxygen deficiency in $\mathrm{WO}_{3}$ leads to a strong increase in conductivity as $\mathrm{W}^{5+}\left(5 d^{1}\right)$ ions are induced, which are a source of the charge carriers. In the octahedral oxygen coordination, $\mathrm{W}^{5+}$ hosts one $5 d$ electron in a triply degenerate orbital and therefore is a Jahn-Teller (JT) ion. A number of experimental studies demonstrated that these charge carriers have a polaronic character and form bipolarons in $\mathrm{WO}_{3-\mathrm{x}}$ [6]. The discovery of high- $T_{\mathrm{c}}$ superconductivity in copper-oxide materials resulted from the concept of JT polarons [7]. Subsequent experimental and theoretical studies proved the validity of this concept by providing ample evidence of polaron/bipolaron formation and their clustering in cuprates [8-11]. Therefore, high- $T_{\mathrm{c}}$ superconductivity may also exist in oxygen-reduced tungsten oxide. Recently, two authors of this paper, Alexander Shengelaya and Kazimierz Conder, together with Alex Müller, reported that in reduced tungsten oxide with the composition $\mathrm{WO}_{2.9}$, the signatures of filamentary superconductivity with $T_{\mathrm{c}}=80 \mathrm{~K}$ can be registered by means of magnetization measurements [12]. By lithium intercalation, the $T_{\mathrm{c}}$ was further increased to $94 \mathrm{~K}$. These results indicate that there is a potential for high- $T_{\mathrm{c}}$ superconductivity in oxygen-reduced tungsten oxide, which has not been sufficiently explored.

Until now, not much was known about the low-temperature transport properties of the $\mathrm{WO}_{2.9}$ phase. A few reports were published where the temperature dependence of resistivity was measured [13-15]. However, all these measurements were performed above liquid nitrogen temperature. Here, we report results of combined magnetic, transport, and magnetotransport measurements of oxygen reduced tungsten oxide $\mathrm{WO}_{2.9}$ in a broad temperature range, including the previously unexplored low temperature $(T<80 \mathrm{~K})$ region.

\section{Experimental Details}

The polycrystalline $\mathrm{WO}_{3-\mathrm{x}}$ samples were prepared by the solid-state reaction method starting from $\mathrm{WO}_{3}$ and $\mathrm{WO}_{2}$ reagents in the powder form. Details on the sample preparation can be found in [12].

The DC magnetization measurements were performed on a Quantum Design magnetic property measurement system (MPMS) magnetometer. For the zero-field-cooled (ZFC) magnetization measurements, the samples were first cooled to $5 \mathrm{~K}$ in a zero magnetic field, then the magnetic field was applied, and the magnetization was measured with increasing temperature.

The transport measurements were performed on a Quantum Design physical property measurement system (PPMS) via a four-probe technique. Electrical contacts were prepared by indium wires and a silver paste. The magnetic field for magnetoresistance measurements was oriented perpendicular to the current direction.

\section{Results and Discussion}

Figure 1 shows the temperature dependence of the $\mathrm{ZFC}$ magnetizations in $\mathrm{WO}_{3-\mathrm{x}}$ samples measured in a magnetic field of 100 Oe. Among $\mathrm{WO}_{3-\mathrm{x}}$ samples with different oxygen contents, only one sample with the composition $\mathrm{WO}_{2.9}$ exhibited a clear decrease in magnetization below $80 \mathrm{~K}$. This diamagnetic transition is characteristic of the superconducting state. As was demonstrated in a previous publication, various results obtained from magnetization measurements strongly support the superconducting nature of the diamagnetic transitions below $T_{\mathrm{C}}=80 \mathrm{~K}$ in reduced tungsten oxide $\mathrm{WO}_{2.9}$ [12]. 


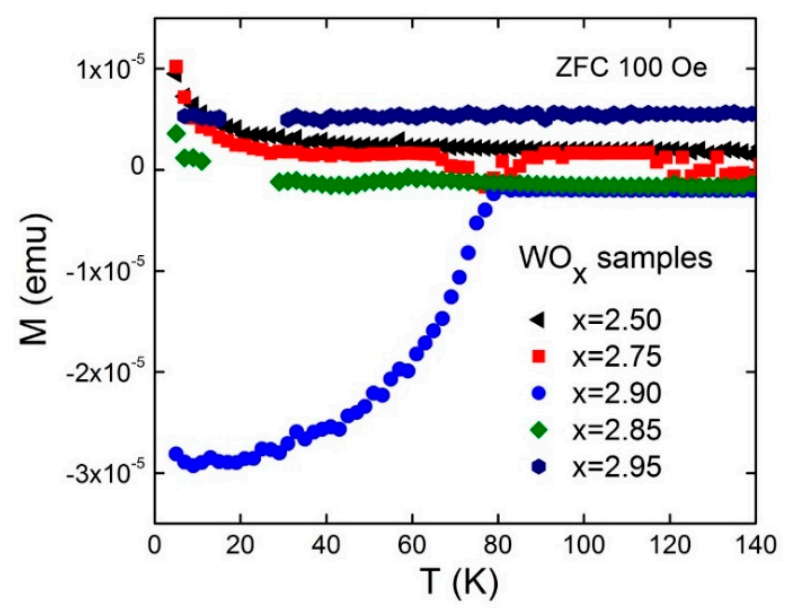

Figure 1. Temperature dependence of the zero-field-cooled (ZFC) magnetizations in a magnetic field of 100 Oe for the $\mathrm{WO}_{3-\mathrm{x}}$ samples with different oxygen contents.

The magnetization data presented in Figure 1 demonstrate that the reduced tungsten oxide $\left(\mathrm{WO}_{2.9}\right)$ is a special case as it has a high-temperature superconducting phase. To understand why this composition is special, recall that the removal of oxygen from $\mathrm{WO}_{3}$ induces polaronic charge carriers and also leads to structural changes. The lattice tends to eliminate single oxygen vacancies by the crystallographic shear (CS) process. As the $\mathrm{x}$ value in $\mathrm{WO}_{3-\mathrm{x}}$ increases, the usual corner-sharing arrangement of octahedra is partially replaced by groups of edge-sharing $\mathrm{WO}_{6}$ octahedra, which form pockets of shear planes [16]. If these shear planes become parallel and equidistant, a crystalline phase with a defined structure arises, as shown by Magnéli [17]. This is the case for the dark blue modification of the tungsten oxide $\mathrm{WO}_{2.9}$, which was the subject of the present study. It crystallizes in a large unit cell containing 20 tungsten and 58 oxygens atoms. Therefore, its stoichiometry can be represented as $\mathrm{W}_{20} \mathrm{O}_{58}$. It belongs to the family of the Magnéli-type oxides with the general formula $\mathrm{W}_{\mathrm{n}} \mathrm{O}_{3 \mathrm{n}-2}$ [18]. The $\mathrm{W}_{20} \mathrm{O}_{58}$ phase is one of the most stable and ordered members of the $\mathrm{W}_{\mathrm{n}} \mathrm{O}_{3 \mathrm{n}-2}$ homologous series, where blocks of $2 \mathrm{D}$ corner-sharing $\mathrm{WO}_{6}$ octahedra are mutually connected along CS planes formed by groups of six edge-sharing octahedra [17]. This phase also has a very narrow stability range close to the theoretical stoichiometry value $\mathrm{WO}_{2.9}$ with composition limits of $\mathrm{WO}_{2.89}-\mathrm{WO}_{2.92}$ [19]. This well-defined composition of the $\mathrm{W}_{20} \mathrm{O}_{58}$ phase explains why we observed signatures of superconductivity only within very limited oxygen contents in the series of studied $\mathrm{WO}_{3-\mathrm{x}}$ samples.

According to Salje and Güttler, $\mathrm{WO}_{2.9}$ is also a special compound among reduced tungsten oxides since it is on the verge of the insulator to metal transition [15]. However, they only measured resistivity in this compound down to $80 \mathrm{~K}$. To better understand transport properties of $\mathrm{WO}_{2.9}$, we performed resistivity and magnetoresistance measurements in a broad temperature range, including the previously unexplored low temperature region. Figure 2 shows the resistivity as a function of temperature for the $\mathrm{WO}_{2.9}$ sample. Metallic behavior $(d \rho / d T>0)$ was observed in the temperature range $16-230 \mathrm{~K}$. In contrast to magnetization measurements, the resistivity had no anomaly at $T_{\mathrm{c}}=80 \mathrm{~K}$, indicating that the sample had a low superconducting volume fraction. The estimation of the superconducting volume fraction based upon the magnetization measurement shown in Figure 1 is $\sim 0.01 \%$. Notably, this value is a lower limit valid under the assumption that the size of superconducting regions $d$ is larger than the London penetration depth $\lambda$. If $d<<\lambda$, the volume fraction will be larger. Still, the superconducting volume fraction in $\mathrm{WO}_{2.9}$ is small and is apparently below the percolation limit. Therefore, the temperature dependences of magnetization and resistivity could be explained by the formation of filamentary, non-percolative superconducting regions below $T_{\mathrm{C}}=80 \mathrm{~K}$ in Magnéli-phase tungsten oxide $\mathrm{WO}_{2.9}\left(\mathrm{~W}_{20} \mathrm{O}_{58}\right)$. 


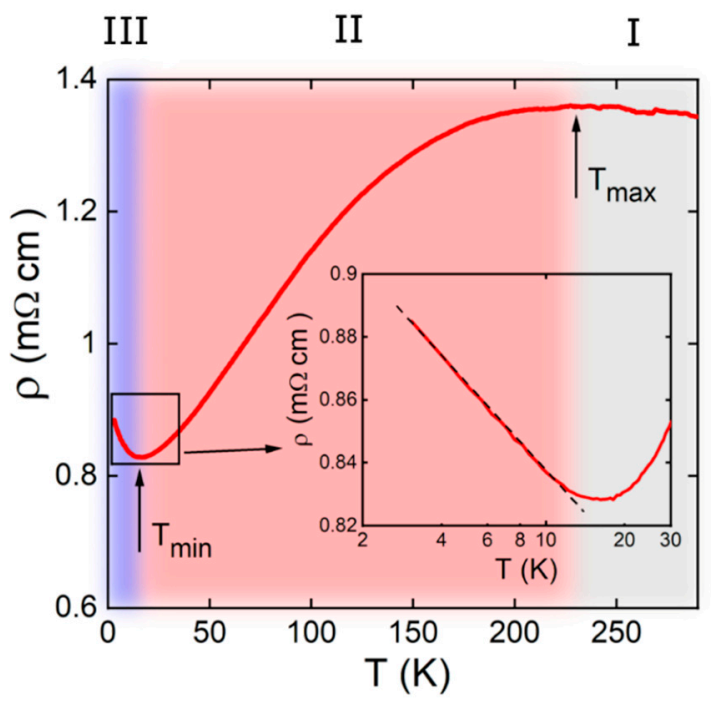

Figure 2. Resistivity as a function of temperature for the $\mathrm{WO}_{2.9}$ sample. Colors indicate temperature regions with three distinct transport regimes. Inset: Expanded view of the low-temperature region shown with a logarithmic horizontal scale. Dashed line is a guide for the eye.

It was proposed that $\mathrm{WO}_{2.9}$ bipolarons can form and cluster in the edge-sharing $\mathrm{WO}_{6}$ octahedra within CS planes of the Magnéli phase [12]. According to this model, CS planes represent charge-carrier-rich quasi-1D stripes. With decreasing temperature, the superconducting state can be established locally in regions similar to cuprates [20]. However, CS planes are very narrow with a thickness of only six edge-sharing $\mathrm{WO}_{6}$ octahedra in the $\mathrm{W}_{20} \mathrm{O}_{58}$ phase. The nanoscale bipolaron-rich metallic regions in CS planes can be locally superconducting, but because of the small size and limited coupling between them, global phase coherence and superconductivity are difficult to establish. Therefore, in the absence of percolation between the CS planes, the superconductivity has a filamentary character as indicated by magnetization and resistivity measurements.

Now, we discuss the temperature dependence of resistivity in more detail, as shown in Figure 2. There are three distinct transport regimes in the $\rho$ (T) dependence: (I) a semiconducting-like behavior above $\sim 230 \mathrm{~K}$ with a negative temperature coefficient, (II) metallic-like behavior in the temperature range of 16-230 K with a positive temperature coefficient, and (III) resistivity upturn below $\sim 16 \mathrm{~K}$.

A broad maximum in the $\rho(T)$ dependence, similar to that shown in Figure 2, was reported previously for $\mathrm{WO}_{2.9}$, but its origin was not discussed [13-15]. Notably, a similar crossover from semiconducting-like conductivity at high temperature to metallic-like behavior at a lower temperature was observed in the normal state of quasi-2D organic superconductors (BEDT-TTF) ${ }_{2} \mathrm{X}\left(\mathrm{ET}_{2} \mathrm{X}\right)$ with $\mathrm{X}=\mathrm{Cu}\left[\mathrm{N}(\mathrm{CN})_{2}\right] \mathrm{Cl}, \mathrm{Cu}\left[\mathrm{N}(\mathrm{CN})_{2}\right] \mathrm{Br}, \mathrm{Cu}(\mathrm{NCS})_{2}$ [21]. Various explanations have been proposed for the different transport regimes and an anomalous resistance maximum [22]. Some models consider the strongly correlated nature of the electrons in organic superconductors. A smooth crossover from an incoherent "bad-metal" state at high temperatures to a coherent Fermi liquid below the resistivity maximum was obtained within the framework of dynamical mean-field theory [17]. Other explanations involve polaron physics to describe anomalous transport behavior. Optical spectroscopy revealed polaron formation in $(\mathrm{ET})_{2} \mathrm{Cu}(\mathrm{NCS})_{2}$ [23]. In particular, the spectra reveal a crossover from a small-polaron-dominated regime at high $T$ to a large-polaron-dominated regime at low $T$. At high temperatures, small polarons do not participate in a coherent motion and the conductivity emerges as an activated hopping of self-trapped carriers. At low temperatures, the average size of polarons increases, and a coherent motion of polarons with resulting metallic behavior appears [24]. Other authors also considered an activated hopping of small polarons at high $T$, but low-temperature metallic behavior was ascribed to tunneling conduction by small polarons according to the prediction of small polaron transport theory [25]. In reduced tungsten oxides, strong electron correlations are not expected, but polaron/bipolaron formation is well documented. Therefore, the observed broad peak in the 
T-dependent resistivity for $\mathrm{WO}_{2.9}$ can be explained as a result of a crossover from high $T$ activated hopping of small (bi)polarons to low $T$ metallic transport due to their tunneling conduction or transition to large (bi)polaron state.

Another notable feature in the $\rho(T)$ dependence of $\mathrm{WO}_{2.9}$ is a resistivity minimum and an upturn toward semiconductor-like behavior, as shown in Figure 2. Interestingly, resistivity increases logarithmically with decreasing temperature, as shown in the inset of Figure 2. Weak localization and the Kondo effect could both lead to this dependence. A logarithmic divergence in the resistivity can be found in Kondo systems, such as dilute magnetic alloys and dense Kondo compounds, where the conduction electrons are scattered by magnetic impurities [26]. However, $\mathrm{WO}_{2.9}$ is clearly not a Kondo system as the presence of magnetic impurities is not supported by our low-temperature magnetization measurements. The logarithmic temperature dependence of the resistivity can also be observed due to the quantum corrections to the resistivity, such as weak localization (WL). WL is a quantum effect caused by constructive interference between the closed-loop trajectories of a diffusive electron. In 2D systems, the WL effect results in a logarithmic rise in resistivity at low temperatures [27]. The WL effect is sensitive to an applied magnetic field, which disturbs the wave coherence and the self-interference effects [28]. Therefore, a magnetic field can destroy WL, which is revealed in experiments as a negative magnetoresistance (MR) and the shift of the resistivity minimum $T_{\min }$ to lower temperatures.

To obtain more information about the unusual transport properties of $\mathrm{WO}_{2.9}$, we performed MR measurements. Figure 3 shows the temperature dependence of resistivity measured in 0 and in $7 \mathrm{~T}$ applied field. $\mathrm{WO}_{2.9}$ exhibits a significant increase in resistivity in a magnetic field in the whole temperature range including the low-temperature upturn. In addition, the resistivity minimum $T_{\min }$ shifts toward higher temperatures from 16 to $23 \mathrm{~K}$ by applying a magnetic field of $7 \mathrm{~T}$. These observations are opposite to what is expected for the WL. Therefore, based on the MR measurements, the WL effect can be excluded in our $\mathrm{WO}_{2.9}$ sample.

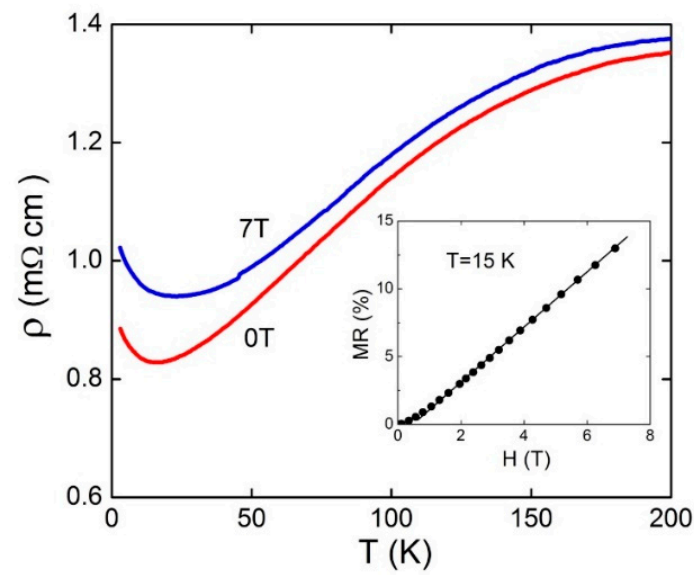

Figure 3. Temperature dependence of resistivity of $\mathrm{WO}_{2.9}$ in zero and in $7 \mathrm{~T}$ applied field. Inset: Magnetic field dependence of magnetoresistance at $T=15 \mathrm{~K}$. Solid line is a guide for the eye.

Notably, a logarithmic low-temperature upturn in the resistivity, similar to that observed here for $\mathrm{WO}_{2.9}$ is a well-known feature of underdoped cuprates [29-31]. It was shown that such behavior can be described remarkably well by resonance Wigner scattering of bipolarons by the random potential in underdoped cuprates [32]. Due to the presence of bipolarons in reduced tungsten oxides, this mechanism provides a very plausible explanation of the observed unusual low-temperature transport properties of $\mathrm{WO}_{2.9}$.

As MR measurements in reduced tungsten oxides have not been reported before, the large positive MR presented in Figure 3 is a new observation. The inset in Figure 3 shows MR as a function of magnetic field at $T=15 \mathrm{~K}$, with the conventional definition $\mathrm{MR}=[[\rho(\mathrm{H})-\rho(0)] / \rho(0)] \times 100 \%$, where $\rho(\mathrm{H})$ and $\rho(0)$ are the values of resistivity at field $H$ and zero, respectively. Above $1 \mathrm{~T}$, MR increases linearly with the 
field without any sign of saturation. Such behavior is incompatible with that expected in conventional metals, where a positive MR arises due to the Lorentz force when the electric and magnetic fields are applied transverse to each other. This mechanism leads to the usual quadratic field dependence of the transverse MR [33]. Even more surprisingly, we found that a positive MR with a similar magnitude also exists in a configuration where a magnetic field was applied parallel to the current direction (longitudinal MR). This isotropic MR excludes the possibility of resulting from the orbital motion of charge carriers. Isotropic MR is usually related to spin scattering mechanisms, but in this case, MR is expected to be negative rather than positive [34]. Therefore, we conclude that the unusual, isotropic, and positive MR observed in $\mathrm{WO}_{2.9}$ at low temperatures cannot be explained by known spin and orbital mechanisms related to charge carriers in a normal state of metals.

Possibly the most interesting aspect of the MR is its strong temperature dependence, which is presented in Figure 4. It shows that at high temperatures, the MR is small and increases very gradually with decreasing temperature. However, it increases strongly upon cooling below $\sim 80 \mathrm{~K}$ and reaches a value of $\sim 15 \%$ at low temperatures in a field of $7 \mathrm{~T}$. This temperature coincides with the superconducting transition observed by magnetization measurements (Figure 1). This suggests that the substantial increase in positive MR below $80 \mathrm{~K}$ could be related to the onset of superconductivity in $\mathrm{WO}_{2.9}$. We note that a considerable positive $\mathrm{MR}$ with unusual temperature dependence was reported recently in strongly underdoped $\mathrm{La}_{2-x} \mathrm{Sr}_{x} \mathrm{CuO}_{4}$ thin films [35]. For example, in a sample where $x=0.05$, a magnetic field of $45 \mathrm{~T}$ caused an excess resistivity of $10 \%$ at a temperature of $10 \mathrm{~K}$. With increasing temperature, the MR decreased to a constant value of $\sim 2 \%$ around $40 \mathrm{~K}$. This temperature corresponds to the maximum $T_{\mathrm{c}}$ observed in optimally doped $\mathrm{La}_{2-\mathrm{x}} \mathrm{Sr}_{\mathrm{x}} \mathrm{CuO}_{4}$. Notably, the sample with $\mathrm{x}=0.05$ did not show signatures of superconductivity in resistivity measurements down to $1.5 \mathrm{~K}$. The considerable excess positive MR observed at low temperatures, which depends non-quadratically on the magnetic field, was attributed to superconducting fluctuations in underdoped $\mathrm{La}_{2-x} \mathrm{Sr}_{x} \mathrm{CuO}_{4}$ [35] . Magnetic field suppresses superconducting fluctuations, leading to an enhanced resistivity. In our opinion, this scenario also applies to the present case and explains the resistivity and MR data obtained in $\mathrm{WO}_{2.9}$. It also accounts for the isotropic character of the observed positive MR in this compound at low temperatures.

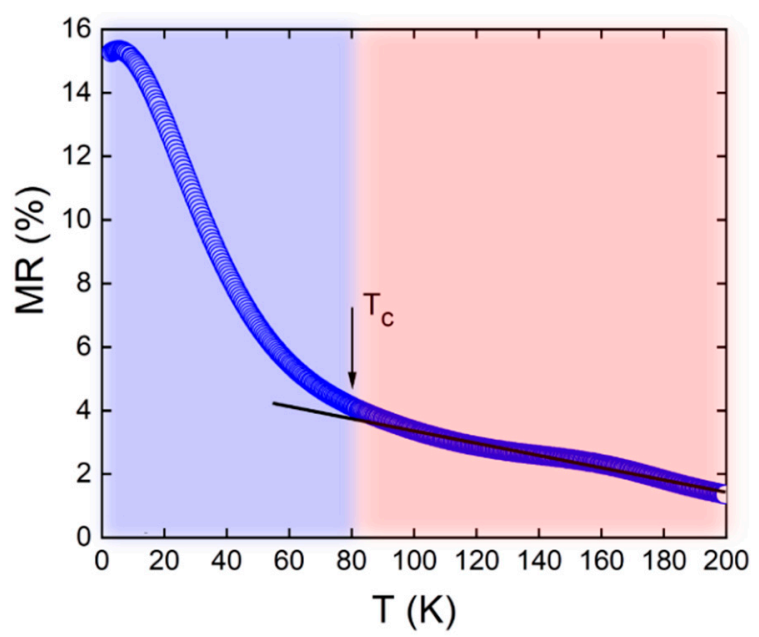

Figure 4. Temperature dependence of magnetoresistance of $\mathrm{WO}_{2.9}$ in $7 \mathrm{~T}$ applied field. The solid line is a guide for the eye. The arrow marks the superconducting transition observed in magnetization measurements.

As discussed above, there are locally-superconducting bipolaron-rich regions in CS planes of $\mathrm{WO}_{2.9}$, but because of the small size and limited coupling between them, global phase coherence and bulk superconductivity are not established. Still, superconducting fluctuations might be present, which are difficult to detect from resistivity measurements. A strong magnetic field would suppress 
superconducting fluctuations; as a result, resistivity increases. The resulting positive MR will have a strong temperature dependence and should disappear above the $T_{\mathrm{C}}$ of superconducting regions. The positive MR detected in this work for $\mathrm{WO}_{2.9}$ and previously in strongly underdoped cuprates seems to be a very sensitive indicator for the presence of small, isolated superconducting clusters embedded in a nonsuperconducting matrix.

\section{Summary and Conclusions}

To summarize, we performed magnetic, transport, and magnetotransport measurements in Magnéli-phase oxygen-reduced tungsten oxide $\mathrm{WO}_{2.9}\left(\mathrm{~W}_{20} \mathrm{O}_{58}\right)$ in a broad temperature range. We found that the temperature dependence of the resistivity is unusual and three different transport regimes exist in $\mathrm{WO}_{2.9}$ : a semiconducting-like behavior above $\sim 230 \mathrm{~K}$ with a negative temperature coefficient, metallic-like behavior in the temperature range of $16-230 \mathrm{~K}$ with a positive temperature coefficient, and resistivity upturn below $\sim 16 \mathrm{~K}$. The broad maximum can be explained as a result of a crossover from high $T$-activated hopping of small (bi)polarons to low- $T$ metallic transport due to either tunneling conduction of small (bi)polarons or transition to large (bi)polaron state.

We observed for the first time that the resistivity of $\mathrm{WO}_{2.9}$ has a minimum with an upturn toward insulator-like behavior below $16 \mathrm{~K}$. We found that the resistivity increases logarithmically with decreasing temperature. MR measurements showed that the observed low-temperature resistivity upturn cannot be explained by known mechanisms, such as weak localization or Kondo effects. One possible explanation of this effect is the resonance Wigner scattering of bipolarons by the random potential in $\mathrm{WO}_{2.9}$. This mechanism was suggested previously for the low-temperature upturn of resistivity in underdoped cuprates.

The superconducting transition at $T_{\mathrm{c}}=80 \mathrm{~K}$ observed in the magnetization measurements was not detected in the temperature dependence of resistivity. This indicates that the superconductivity is localized in small regions that do not percolate. Interestingly, we found a strong increase in positive MR below $80 \mathrm{~K}$. This effect is very similar to that observed in strongly underdoped cuprates, where the considerable excess positive MR was attributed to superconducting fluctuations in small regions. Therefore, we conclude that MR measurements in agreement with magnetization data revealed the presence of superconducting clusters in $\mathrm{WO}_{2.9}$ below $80 \mathrm{~K}$. As suggested in a previous publication, such superconducting clusters form within CS planes that exist in the Magnéli phase of $\mathrm{WO}_{2.9}\left(\mathrm{~W}_{20} \mathrm{O}_{58}\right)$ and represent charge-carrier-rich quasi-1D stripes [12]. These nanoscale clusters can host superconducting pairs, but because of the small size and limited coupling between them, global phase coherence and superconductivity cannot be achieved. Generally, the MR effect is a very sensitive tool for detecting the presence of small, isolated superconducting clusters embedded in a nonsuperconducting matrix.

The obtained results demonstrate that the Magnéli phase of the oxygen-reduced tungsten oxide $\mathrm{WO}_{2.9}\left(\mathrm{~W}_{20} \mathrm{O}_{58}\right)$ has interesting normal and superconducting properties that warrant further studies, especially using single crystal samples. We note that, at present, little is known about the electronic band structure of this material. Recent theoretical studies have suggested that there are several flat bands near the Fermi level [36] and that the electronic properties may be governed by the small number of $d$-bands from the tungsten atoms located in CS planes [37]. So far, experimental studies of the band structure in $\mathrm{WO}_{2.9}$ are lacking.

Especially promising, in our opinion, would be to study pressure effects on the normal and superconducting properties of this compound. By applying hydrostatic or uniaxial pressure, the band structure and coupling between CS planes can be tuned. A well-known example is the ladder compound ( $\mathrm{La}, \mathrm{Sr}, \mathrm{Ca}){ }_{14} \mathrm{Cu}_{24} \mathrm{O}_{41}$, which becomes superconducting under high pressure when the interactions between the ladders are enhanced [38]. Another interesting and novel approach to achieve bulk high- $T_{\mathrm{c}}$ superconductivity in doped tungstates was proposed recently by Müller [39]. Therefore, by improving the coupling and percolation between CS planes, bulk superconductivity and zero resistance state might be achieved in $\mathrm{WO}_{2.9}$. 
Author Contributions: Project planning, A.S.; samples synthesis, K.C.; magnetization measurements, A.S.; transport measurements, F.L.M. and A.S.; writing manuscript, A.S.; review and editing manuscript, A.S., F.L.M., and K.C. All authors have read and agreed to the published version of the manuscript.

Funding: This research was funded by the Swiss National Science Foundation SCOPES Grant No. IZ74Z0-160484 and the Shota Rustavely National Science Foundation of Georgia under grant no. STCU 2017_29.

Acknowledgments: The authors are grateful to K.A. Müller and H. Keller for stimulating discussions.

Conflicts of Interest: The authors declare no conflict of interest.

\section{References}

1. Schooley, J.F.; Hosler, W.R.; Cohen, M.L. Superconductivity in Semiconducting SrTiO3. Phys. Rev. Lett. 1964, 12, 474-475. [CrossRef]

2. Raub, C.J.; Sweedler, A.R.; Jensen, M.A.; Broadston, S.; Matthias, B.T. Superconductivity of Sodium Tungsten Bronzes. Phys. Rev. Lett. 1964, 13, 746-747. [CrossRef]

3. Reich, S.; Tsabba, Y. Possible nucleation of a 2D superconducting phase on WO single crystals surface doped with Na. Eur. Phys. J. B 1999, 9, 1-4. [CrossRef]

4. Shengelaya, A.; Reich, S.; Tsabba, Y.; Müller, K. Electron spin resonance and magnetic susceptibility suggest superconductivity in Na doped WO samples. Eur. Phys. J. B 1999, 12, 13-15. [CrossRef]

5. Aird, A.; Salje, E.K.H. Sheet superconductivity in twin walls: Experimental evidence of. J. Phys. Condens. Matter 1998, 10, L377-L380. [CrossRef]

6. Salje, E.K.H. Polarons and bipolarons in tungsten oxide, $\mathrm{WO}_{3-\mathrm{x}}$. Eur. J. Solid State Inorg. Chem. 1994, 31, 805-821.

7. Bednorz, J.G.; Müller, K.A. Perovskite-Type Oxides-the New Approach to High-TcSuperconductivity. Nobel Lecture. Angew. Chem. Int. Ed. 1988, 27, 735-748. [CrossRef]

8. Bussmann-Holder, A.; Keller, H.; Müller, K.A. Evidences for Polaron Formation in Cuprates. In Family Medicine; Springer Science and Business Media LLC: Berlin/Heidelberg, Germany, 2005; pp. 365-384.

9. Müller, K.A. Essential Heterogeneities in Hole-Doped Cuprate Superconductors. In Family Medicine; Springer Science and Business Media LLC: Berlin/Heidelberg, Germany, 2005; pp. 1-11.

10. Innocenti, D.; Ricci, A.; Poccia, N.; Campi, G.; Fratini, M.; Bianconi, A. A Model for Liquid-Striped Liquid Phase Separation in Liquids of Anisotropic Polarons. J. Supercond. Nov. Magn. 2009, 22, 529-533. [CrossRef]

11. Müller, K.A. The Polaronic Basis for High-Temperature Superconductivity. J. Supercond. Nov. Magn. 2017, 30, 3007-3018. [CrossRef]

12. Shengelaya, A.; Conder, K.; Müller, K.A. Signatures of Filamentary Superconductivity up to 94 K in Tungsten Oxide $\mathrm{WO}_{2.90}$. J. Supercond. Nov. Magn. 2019, 33, 301-306. [CrossRef]

13. Berak, J.M.; Sienko, M. Effect of oxygen-deficiency on electrical transport properties of tungsten trioxide crystals. J. Solid State Chem. 1970, 2, 109-133. [CrossRef]

14. Sahle, W.; Nygren, M. Electrical Conductivity and High Resolution Electron Microscopy Studies of $\mathrm{WO}_{3-\mathrm{x}}$ Crystals with $0 \leq \mathrm{x} \leq 0.28$. J. Solid State Chem. 1983, 48, 154-160. [CrossRef]

15. Salje, E.; Güttler, B. Anderson transition and intermediate polaron formation in $\mathrm{WO}_{3-\mathrm{x}}$ Transport properties and optical absorption. Philos. Mag. B 1984, 50, 607-620. [CrossRef]

16. Tilley, R. The crystal chemistry of the higher tungsten oxides. Int. J. Refract. Met. Hard Mater. 1995, 13, 93-109. [CrossRef]

17. Magnéli, A. Crystal structure studies on beta-tungsten oxide. Arkiv. Kemi 1949, 1, 513-523.

18. Bursill, L.; Hyde, B. CS families derived from the $\mathrm{ReO}_{3}$ structure type: An electron microscope study of reduced $\mathrm{WO}_{3}$ and related pseudobinary systems. J. Solid State Chem. 1972, 4, 430-446. [CrossRef]

19. Marucco, J.-F.; Gerdanian, P.; Dodé, M. Contribution à l'étude thermodynamique des oxydes de tungstène, à $1000{ }^{\circ} \mathrm{C}$, dans le domaine [math]. J. Chim. Phys. 1969, 66, 674-684. [CrossRef]

20. Shengelaya, A.; Müller, K.A. The intrinsic heterogeneity of superconductivity in the cuprates. EPL Europhys. Lett. 2014, 109, 27001. [CrossRef]

21. Ishiguro, T.; Ito, H. Structure and Phase Diagram of Organic Superconductors; Springer Science and Business Media LLC: Berlin/Heidelberg, Germany, 1998; pp. 135-146.

22. Lang, M.; Müller, J. The Physics of Superconductors Vol. II; Bennemann, K.H., Ketterson, J.B., Eds.; Springer: Berlin/Heidelberg, Germany, 2004; pp. 453-554. 
23. Merino, J.; McKenzie, R.H. Transport properties of strongly correlated metals: A dynamical mean-field approach. Phys. Rev. B 2000, 61, 7996-8008. [CrossRef]

24. Wang, N.L.; Clayman, B.P.; Mori, H.; Tanaka, S. Far-infrared study of the insulator-metal transition in kappa-(BEDT-TTF) ${ }_{2} \mathrm{Cu}(\mathrm{NCS})_{2}$ : Evidence for polaron absorption. J. Phys. Condens. Matter 2000, 12, 2867-2875. [CrossRef]

25. Cariss, C.; Porter, L.; Thorn, R. Temperature dependence of conductivity of $\mathrm{k}(\mathrm{BEDT} \mathrm{TTF})_{2} \mathrm{Cu}(\mathrm{SCN})_{2}$; Resolution into two components; Small polaron. Solid State Commun. 1990, 74, 1269-1273. [CrossRef]

26. Kondo, J. Resistance Minimum in Dilute Magnetic Alloys. Prog. Theor. Phys. 1964, 32, 37-49. [CrossRef]

27. Abrahams, E.; Anderson, P.W.; Licciardello, D.C.; Ramakrishnan, T.V. Scaling Theory of Localization: Absence of Quantum Diffusion in Two Dimensions. Phys. Rev. Lett. 1979, 42, 673-676. [CrossRef]

28. Altshuler, B.L.; Khmel'Nitzkii, D.; Larkin, A.I.; Lee, P.A. Magnetoresistance and Hall effect in a disordered two-dimensional electron gas. Phys. Rev. B 1980, 22, 5142-5153. [CrossRef]

29. Boebinger, G.S.; Ando, Y.; Passner, A.; Kimura, T.; Okuya, M.; Shimoyama, J.; Kishio, K.; Tamasaku, K.; Ichikawa, N.; Uchida, S. nsulator-to-Metal Crossover in the Normal State of $\mathrm{La}_{2-\mathrm{x}} \mathrm{Sr}_{\mathrm{x}} \mathrm{CuO}_{4}$ Near Optimum Doping. Phys. Rev. Lett. 1996, 77, 5417-5420. [CrossRef]

30. Ando, Y.; Boebinger, G.S.; Passner, A.; Kimura, T.; Kishio, K. Logarithmic Divergence of both In-Plane and Out-of-Plane Normal-State Resistivities of Superconducting $\mathrm{La}_{2-\mathrm{x}} \mathrm{Sr}_{\mathrm{x}} \mathrm{CuO}_{4}$ in the Zero-Temperature Limit. Phys. Rev. Lett. 1995, 75, 4662-4665. [CrossRef]

31. Fournier, P.; Mohanty, P.; Maiser, E.; Darzens, S.; Venkatesan, T.; Lobb, C.J.; Czjzek, G.; Webb, R.A.; Greene, R.L. Insulator-Metal Crossover near Optimal Doping in $\operatorname{Pr}_{2-x} \mathrm{Ce}_{\mathrm{x}} \mathrm{CuO}_{4}$ : Anomalous Normal-State Low Temperature Resistivity. Phys. Rev. Lett. 1998, 81, 4720-4723. [CrossRef]

32. Alexandrov, A. Logarithmic normal state resistivity of high-Tc cuprates. Phys. Lett. A 1997, 236, $132-136$. [CrossRef]

33. Pippard, A.B. Magnetoresistance in Metals; Cambridge University Press: Cambridge, UK, 1989.

34. Preyer, N.W.; Kastner, M.A.; Chen, C.Y.; Birgeneau, R.J.; Hidaka, Y. Isotropic negative magnetoresistance in $\mathrm{La}_{2-\mathrm{x}} \mathrm{Sr}_{\mathrm{x}} \mathrm{CuO}_{4+\mathrm{y}}$. Phys. Rev. B 1991, 44, 407-410. [CrossRef]

35. Vanacken, J.; Moshchalkov, V.V. Transport properties of high-Tc cuprate thin films as superconductive materials. In High-Temperature Superconductors; Qiu, X.G., Ed.; Woodhead Publishing: Cambridge, UK, 2011; pp. 38-102.

36. Sun, L.; Li, Z.; Su, R.; Wang, Y.; Li, Z.; Du, B.; Sun, Y.; Guan, P.; Besenbacher, F.; Yu, M. Phase-Transition Induced Conversion into a Photothermal Material: Quasi-Metallic $\mathrm{WO}_{2.9}$ Nanorods for Solar Water Evaporation and Anticancer Photothermal Therapy. Angew. Chem. Int. Ed. 2018, 57, 10666-10671. [CrossRef]

37. Slobodchikov, A.A.; Nekrasov, I.A.; Pavlov, N.S.; Korshunov, M.M. From the open Heisenberg model to the Landau-Lifshitz equation. arXiv 2020, arXiv:2006.16658v1.

38. Uehara, M.; Nagata, T.; Akimitsu, J.; Takahashi, H.; Mori, N.; Kinoshita, K. Superconductivity in the Ladder Material $\mathrm{Sr}_{0.4} \mathrm{Ca}_{13.6} \mathrm{Cu}_{24} \mathrm{O}_{41.84}$. J. Phys. Soc. Jpn. 1996, 65, 2764-2767. [CrossRef]

39. Müller, K.A. Generation of Bulk HTS with Doped Tungstates. J. Supercond. Nov. Magn. 2017, 30, $2707-2709$. [CrossRef]

Publisher's Note: MDPI stays neutral with regard to jurisdictional claims in published maps and institutional affiliations.

(C) 2020 by the authors. Licensee MDPI, Basel, Switzerland. This article is an open access article distributed under the terms and conditions of the Creative Commons Attribution (CC BY) license (http://creativecommons.org/licenses/by/4.0/). 